\section{Food hygiene control in school canteens of La Spezia municipality: years 2003-2012}

\author{
Laura Serracca, ${ }^{1}$ Roberta Battistini, ${ }^{1}$ \\ Irene Rossini, ${ }^{1}$ Marco Imberciadori, ${ }^{1}$ \\ Cristina Sturlese, ${ }^{1}$ Elena Bozzetta, ${ }^{2}$ \\ Carlo Ercolini ${ }^{1}$ \\ 'Istituto Zooprofilattico Sperimentale del \\ Piemonte, Liguria e Valle d'Aosta, \\ Sezione di La Spezia, Italy; 'Istituto \\ Zooprofilattico Sperimentale \\ del Piemonte, Liguria e Valle d'Aosta, \\ Torino, Italy
}

\section{Abstract}

The school canteens are public catering services of great interest as they provide meals to a high number of consumers who are particularly susceptible to health risks, therefore surveillance and health control are very important to ensure food safety. To this purpose, a convention between the Istituto Zooprofilattico Sperimentale of Piemonte Liguria e Valle d'Aosta, and La Spezia municipality was established for the health control of school canteens. In this article we report the results of analysis performed on food and swab surfaces samples collected during the period 2003-2012 in 22 school canteens and 3 cooking centers. From a total of 1187 samples: 313 raw foods were analyzed for chemical and microbiological parameters to verify compliance with legislation, 71 food preparations were analyzed for bacteria indicators to assess the good manufacturing practices, and 803 surface swabs were tested for total mesophilic count (TMC), Salmonella spp. and Listeria spp. to control cleaning/disinfection conditions. The results show that only $1.3 \%$ of raw food samples did not respect the limits imposed by legislation, and $1.4 \%$ of food preparations was positive for pathogens. In environmental swabs, pathogenic microorganisms were never isolated and TMC exceeded the limits of acceptability in no more than $27 \%$ of cases. The most contaminated surfaces were those in contact with food and the equipment difficult to clean. The results demonstrate that potential hazards are kept to acceptable levels in school canteens and cooking centers investigated. In fact, during the period considered no foodborne diseases were reported among users. However, data obtained may be useful to better define control measures to be adopted to improve the hygienic level production in these structures and to prevent foodborne infections.

\section{Introduzione}

La ristorazione scolastica ha subito negli ultimi anni un'enorme diffusione, soprattutto a seguito dell'introduzione del tempo pieno nella scuola dell'obbligo e della sempre maggiore occupazione femminile in attività extradomestiche. L'incremento della produzione e della distribuzione di pasti in questo settore ha portato pertanto come conseguenza un possibile aumento dell'incidenza di tossinfezioni tra la popolazione in età scolare che risulta più suscettibile all'azione di eventuali patogeni presenti negli alimenti (Gerber et al., 1996). I pasti che vengono somministrati nell'ambito della ristorazione scolastica hanno come requisito fondamentale e prioritario quello di non costituire un pericolo per la salute dei consumatori. Particolare attenzione deve pertanto essere posta alle caratteristiche igienico-sanitarie dei cibi somministrati attraverso un attento ed accurato controllo del ciclo produttivo (Ministero della Salute, 2010). Nonostante la continua evoluzione delle tecniche di preparazione e di conservazione degli alimenti ed un efficace piano di controlli sanitari, previsto dalle norme comunitarie e nazionali [Reg. CE n. 178/2002 (Commissione Europea, 2002); Clerici e Rubino, 2004; Reg CE n. 1441/2007 (Commissione Europea, 2007); D. Lgs 193/2007 (Repubblica Italiana, 2007)], la ristorazione collettiva non è del tutto esente da rischi, tanto che non sono infrequenti gli episodi di malattie alimentari (EFSA, 2012). L'incidenza delle malattie a trasmissione alimentare (MTA) o tossinfezioni alimentari è in costante ascesa in tutti i Paesi industrializzati, rappresentando ancora un forte impatto sulla salute della collettività (0'Brien, 2012), sebbene lo scenario epidemiologico delle tossinfezioni alimentari nell'ultimo decennio abbia subito profondi cambiamenti, dovuti sia all'emergenza di nuovi agenti patogeni (quali E. coli produttori di verotossina, e nuovi sierotipi di Salmonella), sia alla descrizione di nuovi veicoli di trasmissione come emerge dall'analisi dei dati di notifica di MTA dal 1998 e fino al 2009 (Ministero della Salute, 2011). Tra i fattori di rischio che contribuiscono all'insorgenza di MTA nell'ambito della ristorazione collettiva abbiamo: il mancato rispetto dell'osservanza delle norme igieniche durante le fasi di preparazione, l'insufficiente sanificazione delle attrezzature e degli utensili, la contaminazione crociata tra alimenti crudi e cotti, le inadeguate temperature e/o tempi di cottura, la conservazione degli alimenti cotti a temperature non adeguate e temperature di refrigerazione non adeguate (Schaffner, 2008). In considerazione dell'elevato numero di consumatori e dell'importanza di queste strutture da un punto di vista sanitario risulta quindi evidente l'importanza della vigilanza e del con-
Correspondence: Laura Serracca, Istituto Zooprofilattico Sperimentale del Piemonte, Liguria e Valle d'Aosta, via degli Stagnoni 96, 19100 La Spezia, Italy.

Tel. +39.0187.507370 - Fax: +39.0187 .500308 .

E-mail: laura.serracca@izsto.it

Key words: School canteen, Microbiological analysis, Food, Swab, Surface.

Conflict of interests: the authors declare no potential conflict of interests.

Received for publication: 15 January 2013.

Revision received: 27 February 2013.

Accepted for publication: 1 March 2013.

This work is licensed under a Creative Commons Attribution 3.0 License (by-nc 3.0).

(C) Copyright L.Serracca et al., 2013

Licensee PAGEPress, Italy

Italian Journal of Food Safety 2013; 2:e11

doi:10.4081/ijfs.2013.e11

trollo nella ristorazione scolastica, che, per risultare efficaci, devono essere effettuati con metodo e rigore, secondo programmi preordinati, così da prevenire l'instaurarsi di fattori di rischio e garantire la sicurezza alimentare. Nel presente lavoro sono riportati i risultati delle analisi microbiologiche e chimiche eseguite su campioni di materie prime, tamponi ambientali e pasti, prodotti e/o distribuiti nelle mense scolastiche del comune di La Spezia relativi agli ultimi 10 anni. Tali dati scaturiscono a seguito di una convenzione stipulata tra il comune suddetto e l'Istituto Zooprofilattico Sperimentale del Piemonte Liguria e Valle d'Aosta (IZS PLVA) allo scopo di verificare la rispondenza ai parametri igienico sanitari, previsti dalla normativa vigente, degli alimenti prodotti e consumati in tali esercizi.

\section{Materiali e Metodi}

L'indagine è stata condotta su 22 mense scolastiche (6 asili nido, 7 scuole materne e dal 2010 i controlli sono stati estesi anche a 4 scuole elementari e 5 medie) e 3 centri di cottura presenti nel comune di La Spezia che producono e distribuiscono pasti a bambini di età scolare e prescolare. Tali strutture sono state monitorate a partire dall'anno 2003 e fino a giugno 2012, periodo di tempo in cui sono stati prelevati un totale di 1187 campioni così suddivisi: 313 materie prime, 71 preparazioni alimentari e 803 tamponi ambientali. Il prelievo dei campioni è stato effettuato dal personale tecnico del comune e i campioni sono stati trasportati al laboratorio di analisi in idonei contenitori refrigerati $\left(4 \pm 3^{\circ} \mathrm{C}\right)$, mentre la parte 
analitica è stata effettuata dal personale tecnico dell'IZS Sezione di La Spezia. Per verificare la conformità delle materie prime e degli alimenti somministrati alla normativa vigente, sono stati ricercati i parametri chimici e microbiologici previsti dal Reg. CE 466/2001 (Commissione Europea, 2001) e successive modifiche e dal Regolamento CE 1441/2007 (Commissione Europea, 2007), nonché dal Piano Nazionale Residui in applicazione del D. Lgs 336/99 (Repubblica Italiana, 1999) ed ai relativi limiti forniti dalla normativa stessa. Per verificare la corretta applicazione delle buone pratiche di lavorazione, negli alimenti prodotti è stata effettuata una valutazione quantitativa di batteri indicatori particolarmente sensibili a tali pratiche [Escherichia coli (E. coli); carica batterica totale (CBT); coliformi; stafilococchi coagulasi positivi; Salmonella spp.; Listeria spp.; anaerobi solfito riduttori; Bacillus cereus (B. cereus)], per cui la loro presenza nel campione in quantità superiore ai limiti definiti dalla letteratura scientifica, indica che sono stati commessi errori o mancanze nelle pratiche di produzione, conservazione e distribuzione del pasto. Infine, per verificare l'efficacia delle operazioni di detersione/disinfezione è stata ricercata la carica mesofila totale (CMT) come indicatore di contaminazione globale, prelevata tramite tampone da superfici di $10 \mathrm{~cm}^{2}$, sulle superfici di lavoro, sulle attrezzature e sulle mani degli operatori. Per le attrezzature di difficile pulizia, alla ricerca dei patogeni ubiquitari quali Salmonella spp. e Listeria monocytogenes, è stata associata anche quella della CMT a partire dal 2009, mentre la CMT sulle mani degli operatori è stata rilevata fino al 2006. Le metodiche analitiche utilizzate per la ricerca dei vari parametri sono riportate in Tabella 1 , mentre i limiti microbiologici di accettabilità per verificare la corretta sanitizzazione delle superfici e delle attrezzature in base alla carica batterica presente, sono riportati in Tabella 2 ; tali valori fanno riferimento agli standard proposti in letteratura (INAIL, 2005).

\section{Risultati}

\section{Materie prime e/o alimenti somministrati tali quali}

In totale nel periodo indagato su 313 campioni di materie prime e/o alimenti somministrati tali quali sono state registrate solamente 4 non conformità (1,3\%). In particolare nel 2003 sono risultati non conformi 2 campioni di pesce, uno congelato ed uno refrigerato, per la presenza di mercurio in quantità di 1,6 e 2,66 $\mathrm{mg} / \mathrm{Kg}$ rispettivamente, e quindi superiore al limite massimo consentito pari a $1,0 \mathrm{mg} / \mathrm{Kg}$ secondo la normativa in vigore al momento dell'analisi (Reg. CEE n. 466/2001; Commis- sione Europea, 2001). Nel 2005 e nel 2010 si sono invece avuti due campioni in cui la carne di suino ha dato esito positivo per il patogeno Salmonella spp. (presenza in $25 \mathrm{~g}$ ) (ISO, 2004a).

\section{Preparazioni alimentari}

Tra i campioni di alimenti prodotti o manipolati nelle strutture campionate è risultato non conforme solamente 1 campione su 71 $(1,4 \%)$ : una preparazione gastronomica ready to eat di riso freddo positiva per B. cereus $(2000$ UFC/g) (ISO, 2004b).

\section{Tamponi ambientali}

Superfici non a contatto con alimenti (pareti di celle frigo, pareti armadi, ripiani armadi, ripiani carrelli, tavoli mense, porte, porte frigoriferi, maniglie, rubinetti, piastrelle pareti): su un totale di 208 tamponi solamente $8(3,8 \%)$ hanno superato i limiti di accettabilità previsti. Le superfici più contaminate sono state le pareti delle celle frigo.

Superfici a contatto con alimenti (piani di lavoro, taglieri, teglie, vassoi contenitori in acciaio, gastronorm, piatti, vassoi self-service, coltelli, mestoli, palette, pentole, pinze): per questo tipo di superfici le non conformità sono state in media del $16 \%$ circa (67/414) anche se sono risultate più frequenti negli anni dal 2003 al 2006. Il superamento dei limiti di accettabilità si è verificato in massima parte su taglieri, gastronorm, mestoli e vassoi del self-service.

Personale: i dati riguardanti la verifica dell'igiene, effettuata con tamponi sulle mani, hanno evidenziato nel 7,24\% dei casi il superamento dei limiti di accettabilità per la CMT su un totale di 138 determinazioni analitiche. Le non conformità si sono avute nei primi due anni considerati ed in particolare nel 2004.

Attrezzature di difficile pulizia (affettatrici, polpettatrice, tritacarne, frullatore, taglieri di legno, tritaverdure, tritatutto, grattugie, spre-

Tabella 1. Metodi utilizzati durante le analisi.

\begin{tabular}{|c|c|}
\hline Parametro & Metodo di analisi \\
\hline E. coli & ISO 16649-2:2001 (ISO, 2001) \\
\hline CBT & ISO 4833:2003 (ISO, 2003b) \\
\hline Coliformi & ISO 4832:2006 (ISO, 2006); ISO 7218:2007 (ISO, 2007a) \\
\hline Stafilococchi coagulasi positivi & ISO 6888-2:1999/Amend 1:2003 (ISO, 2003a) \\
\hline Salmonella spp. & AFNOR V08-052:1997 (AFNOR, 1997); AFNOR BIO 12/10-09/02 (AFNOR, 2006) \\
\hline Anaerobi solfito riduttori & ISO 15213:2003 (ISO, 2003c) \\
\hline Listeria spp. & ISO 11290-2:1998/Amd 1:2004 (ISO, 2004c) \\
\hline B. cereus & ISO 7932:2004 (ISO, 2004b) \\
\hline CMT & ISO 4833:2003 (ISO, 2003b) \\
\hline L. monocytogenes & AFNOR BIO 12/11-03/04 OM 07/12/1993 G.U. n 291 13/12/1993 (AFNOR, 2004) \\
\hline Aflatossine & HPLC-FLD; ELISA \\
\hline E. coli 0157 & AFNOR BIO 12/8-07/00 (AFNOR, 2000) \\
\hline Additivi & D.M. 27/02/1996 n²09 - G.U. n96 (Ministero della Salute, 1996) \\
\hline Inibenti & AFNOR DSM 28/1-06/06 (AFNOR, 2006) \\
\hline Istamina & HPLC \\
\hline V. cholerae & ISO/TS 21872:2007 (ISO, 2007b) \\
\hline V. parahaemolyticus & ISO/TS 21872:2007 (ISO, 2007b) \\
\hline $\mathrm{Pb}$ & Rapporto ISTISAN 96/34 (Istituto Superiore di Sanità, 1996) \\
\hline $\mathrm{Cd}$ & Rapporto ISTISAN 96/34 (Istituto Superiore di Sanità, 1996) \\
\hline $\mathrm{Hg}$ & D.M. 14/12/1971 (Ministero della Salute, 1971) \\
\hline Fitosanitari & HPLC-DAD; LC/MS/MS \\
\hline OGM & PCR; CTAB (ISO 21571:2005) (ISO, 2005) \\
\hline TVN & Titolazione acido-base \\
\hline DES & ELISA \\
\hline Anabolizzanti & ELISA \\
\hline Antiparassitario & HPLC-DAD; LC/MS/MS \\
\hline \multicolumn{2}{|c|}{$\begin{array}{l}\text { E. coli, Escherichia coli; CBT, carica batterica totale; Salmonella spp., Salmonella species; Listeria spp., Listeria species; B. cereus, Bacillus } \\
\text { cereus; CMT, carica mesofila totale; L. monocytogenes, Listeria monocytogenes; V. cholerae, Vibrio cholerae; V. parahaemolyticus, Vibrio para- } \\
\text { haemolyticus; Pb, piombo; Cd, cadmio; Hg, mercurio; OGM, organismi geneticamente modificati; TVN, total volatile nitrogen (i.e. azoto volatile } \\
\text { totale); DES, diethylstillestrol (i.e. ormoni di sintesi); HPLC-FLD, high performance liquid chromatography with postcolumn fluorescence } \\
\text { derivatization; ELISA, enzyme-linked immuno sorbent assay test; HPLC, high performance liquid chromatography-mass spectrometry; HPLC- } \\
\text { DAD, high performance liquid chromatography with diode aray detector, LC/MS/MS, liquid chromatography-tandem mass spectrometry; PCR, }\end{array}$} \\
\hline
\end{tabular}


miagrumi): tutti i tamponi eseguiti su queste superfici (particolarmente a rischio) hanno dato esito negativo per la ricerca dei patogeni Salmonella spp. e $L$. monocytogenes nei 10 anni considerati, mentre la ricerca della CMT, effettuata a partire dal 2009, è risultata al di sopra dei limiti in 8 tamponi su $43(18,6 \%)$. Polpettatrice e taglieri di legno sono le superfici che più spesso hanno superato i limiti di accettabilità.

Analizzando le percentuali complessive delle CMT al di sopra del limite di accettabilità nelle diverse strutture campionate (Figura 1), risulta che le mense delle scuole elementari sono quelle che hanno dato maggiori non conformità (25\%), anche se lo scarso numero di tamponi effettuati (4) rende il campione poco significativo. Seguono con percentuali simili scuole materne e centri cottura con il 13,5 ed il $12,8 \%$ rispettivamente, mentre asili nido e scuole medie presentano le percentuali più basse, $8,7 \%$ i primi ed $8 \%$ le seconde. Se analizziamo invece l'andamento del superamento dei limiti nei diversi anni (Figura 2) possiamo notare come i dati siano molto variabili e le strutture non presentino né una tendenza netta al miglioramento né al peggioramento nel tempo.

Tabella 2. Classificazione delle superfici, delle attrezzature e delle mani degli operatori in base alla carica batterica presente.

\begin{tabular}{lc} 
Carica batterica & Giudivio \\
$\mathrm{CMT}<10 \mathrm{UFC} / \mathrm{cm}^{2}$ & Buono \\
$\mathrm{CMT}=11-50 \mathrm{UFC} / \mathrm{cm}^{2}$ & Accettabile \\
\hline $\mathrm{CMT}>50 \mathrm{UFC} / \mathrm{cm}^{2}$ & Insufficiente \\
\hline
\end{tabular}

CMT, carica mesofila totale.

\section{Discussione}

Le materie prime utilizzate risultano in generale di buona qualità infatti in 10 anni di studio si sono registrate solamente 4 non conformità che hanno riguardato alimenti di origine animale (pesce e carni di suino). Per quanto riguarda le carni di suino, la presenza di Salmonella non rappresenta un grave rischio per il target della ristorazione collettiva essendo abolita, in tale comparto, la preparazione di piatti costituiti da carne cruda o poco cotta (Doyle e Mazzotta, 2000). Risulta importante però la corretta applicazione di una good manufacturing practice (GMP), per quanto riguarda lo stoccaggio intermedio, al fine di evitare contaminazioni crociate tra cibi crudi e cotti (Bertoldi e Galli, 2012). La presenza di mercurio, in concentrazione superiore al limite consentito nel pesce congelato, non comporta interventi di tipo sanitario ma piuttosto verifiche sui fornitori. Gli alimenti somministrati tali quali sono invece sempre stati conformi ai parametri di legge.

Per quanto riguarda le preparazioni alimentari la presenza di 1 sola non conformità dimostra la corretta applicazione delle buone pratiche di lavorazione da parte degli operatori nelle strutture campionate. Il ritrovamento di $B$. cereus nella preparazione gastronomica di riso freddo risultata positiva potrebbe essere imputata alla preparazione con largo anticipo rispetto al consumo, unita alla conservazione errata del prodotto 0 all'aggiunta di ingredienti crudi dopo la cottura con conseguente contaminazione e crescita del microrganismo (Cantoni et al., 2002).

Dai dati relativi ai tamponi ambientali emerge che le superfici più a rischio per il superamento dei limiti sono quelle a contatto con gli alimenti seguite dalle attrezzature di difficile pulizia dove tuttavia non sono mai stati ritrovati microrganismi patogeni. Per quest'ultime superfici il riscontro di non conformità soprattutto su polpettatrici e taglieri di legno evidenza la difficoltà nel sanificare correttamente questi strumenti, risulta pertanto opportuno rivedere i piani di pulizia e disinfezione, e formare e sensibilizzare ulteriormente il personale perché ponga maggiore attenzione alla sanificazione di queste superfici (Cappelli, 2008). Nel caso invece delle piccole attrezzature e delle superfici non a contatto con gli alimenti, le percentuali di non conformità sono molto basse evidenziando in generale la corretta esecuzione delle procedure di pulizia e disinfezione adottate. Tuttavia, al fine di un miglioramento costante è opportuno da parte del personale porre ancora più attenzione 0 incrementare la frequenza di pulizia delle pareti delle celle frigorifero, dei mestoli e dei vassoi del self-service in modo da ridurre le condizioni che possano favorire lo sviluppo del pericolo (Marriott e Gravani, 2008).

Le cariche batteriche riscontrate sulle mani degli operatori hanno fatto registrare solo il $7,24 \%$ di non conformità negli anni 2003 e 2004 per poi migliorare raggiungendo valori sempre accettabili negli anni successivi. Questi risultati dimostrano quindi la buona conoscenza e applicazione delle procedure operative da parte degli operatori e la validità dei corsi di formazione effettuati per sensibilizzare la loro igiene personale.

\section{Conclusioni}

In generale la contaminazione globale è risultata bassa in tutte le strutture campionate nel corso dei dieci anni indagati dimostrando che i potenziali pericoli sono mantenuti a livelli pressoché accettabili nella maggior parte delle mense e centri cottura oggetto di indagi-

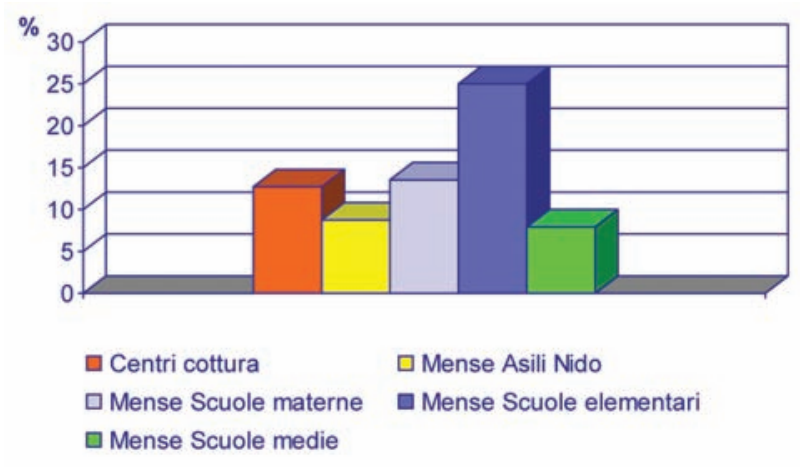

Figura 1. Tamponi ambientali: percentuale della carica mesofila totale al di sopra del limite di accettabilità nelle varie strutture, calcolata sul totale dei campioni analizzati nei 10 anni (20032012).

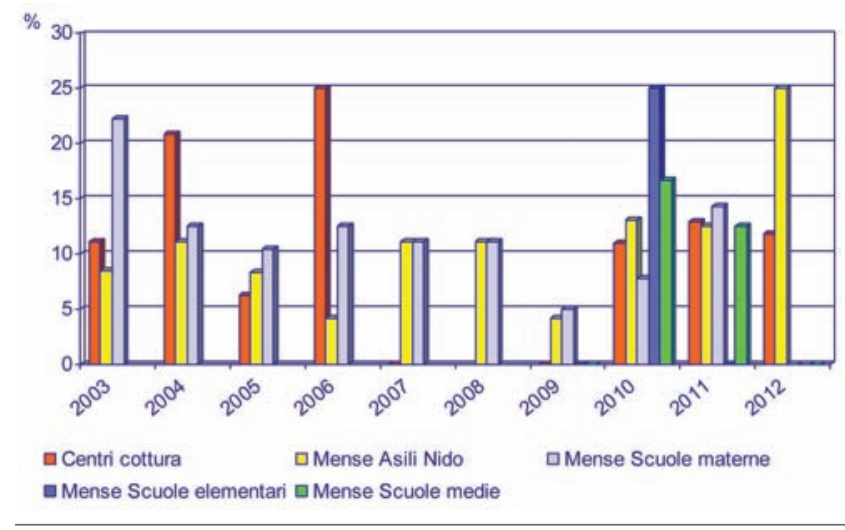

Figura 2. Tamponi ambientali: percentuale della carica mesofila totale al di sopra del limite di accettabilità nelle varie strutture per ogni anno analizzato. 
ne; durante il periodo considerato, infatti, non vi sono stati casi segnalati di tossinfezioni alimentari fra l'utenza. Tuttavia, l'andamento altalenante del superamento dei limiti indica che alcune problematiche non sono ancora completamente sotto controllo. In un'ottica di miglioramento continuo, pertanto, il costante monitoraggio analitico e la continua formazione del personale rappresentano degli strumenti validi per indagare sui fattori responsabili del superamento dei limiti microbiologici ed intervenire in modo efficace e mirato, contribuendo così ad elevare sempre di più nel tempo il livello igienico della produzione (Zacconi et al., 2001; Di Luch, 2002).

\section{Bibliografia}

AFNOR, 1997. Certificat V08-052:1997. Association Française de Normalisation ed., La Plaine Saint-Denis, Francia.

AFNOR, 2000. Certificat BIO 12/8-07/00. Association Française de Normalisation ed., La Plaine Saint-Denis, Francia.

AFNOR, 2002. Certificat BIO 12/10-09/02. Association Française de Normalisation ed., La Plaine Saint-Denis, Francia.

AFNOR, 2004. Certificat BI0 12/11-03/04. Association Française de Normalisation ed., La Plaine Saint-Denis, Francia.

AFNOR, 2006. Certificat DSM 28/1-06/06. Association Française de Normalisation ed., La Plaine Saint-Denis, Francia.

Bertoldi A, Galli A, 2012. Igiene degli alimenti e HACCP. In accordo con le disposizioni del pacchetto igiene modelli applicativi. V edizione. EPC ed., Roma, Italia.

Cantoni CA, Stella S, Cocolin L, Comi G, Marchese R, Ripamonti B, 2002. Identificazione di ceppi di Bacillus spp. isolati da varie tipologie di alimenti. Ind Aliment-Italy 40:268-73.

Cappelli M, 2008. La formazione igienico-sanitaria degli operatori e addetti del settore alimentare. Parte terza. Eurocarni 4:19921.

Clerici A, Rubino V, 2005. La nuova disciplina comunitaria sull'igiene delle produzioni alimentari. Pacchetto Igiene 2004. Taro ed., Alessandria, Italia.

Commissione Europea, 2001. Regolamento della Commissione europea dell'8 marzo 2001 che definisce i tenori massimi di taluni contaminanti presenti nelle derrate alimentari, 466/2001/CE. In: Gazzetta Ufficiale, L 77/1, 16/03/2001.

Commissione Europea, 2002. Regolamento del parlamento europeo e del consiglio del 28 gennaio 2002 che stabilisce i principi e i requisiti generali della legislazione alimentare, istituisce l'Autorità europea per la sicurezza alimentare e fissa procedure nel campo della sicurezza alimentare, 178/2002/CEE. In: Gazzetta Ufficiale, L 31/1, 01/02/2002.

Commissione Europea, 2007. Regolamento della commissione del 5 dicembre 2007 che modifica il regolamento (CE) $n$. 2073/2005 sui criteri microbiologici applicabili ai prodotti, 1441/2007/CE. In: Gazzetta Ufficiale, L 322/12, 07/12/2007.

Di Luch R, 2002. Valutazione qualitativa del rischio nella ristorazione scolastica. Ind Aliment-Italy 41:1295-01.

Doyle ME, Mazzotta AS, 2000. Review of studies on the thermal resistance of Salmonellae. J Food Protect 63:779-95.

EFSA, 2012. The European Union Summary Report on Trends and Sources of Zoonoses, Zoonotic Agents and Foodborne Outbreaks in 2010. EFSA Journal 10:2597. Disponibile al sito: http://www. efsa.europa.eu/en/search.htm?text=EFSA +Journal+2012\%3B+10\%283\%29\%3A2597

Gerber CP, Rose JB, Haas CN, 1996. Sensitive populations. Who is at the greatest risk? Int J Food Microbiol 30:112-23.

INAIL, 2005. Il monitoraggio microbiologico degli ambienti di lavoro. Campionamento e analisi. Istituto Nazionale per l'Assicurazione contro gli Infortuni sul Lavoro ed., Roma, Italia.

ISO, 2001. Microbiology of food and animal feeding stuffs. Horizontal method for the enumeration of beta-glucuronidase-positive Escherichia coli. Part 2: colony-count technique at 44 degrees $\mathrm{C}$ using 5-bromo4-chloro-3-indolyl beta-D-glucuronide. Norma ISO 16649-2:2001. Organizzazione internazionale per la normazione ed., Ginevra, Svizzera.

ISO, 2003a. Microbiology of food and animal feeding stuffs. Horizontal method for the enumeration of coagulase-positive staphylococci (Staphylococcus aureus and other species). Part 2: Technique using rabbit plasma fibrinogen agar medium. Including amendment 1 2003. Norma ISO 68882:1999/Amend 1:2003. Organizzazione internazionale per la normazione ed., Ginevra, Svizzera.

ISO, 2003b. Microbiology of food and animal feeding stuffs. Horizontal method for the enumeration of microorganisms. Colonycount technique at 30 degrees $\mathrm{C}$. Norma ISO 4833:2003. Organizzazione internazionale per la normazione ed., Ginevra, Svizzera.

ISO, 2003c. Microbiology of food and animal feeding stuffs. Horizontal method for the enumeration of sulfite-reducing bacteria growing under anaerobic conditions. Norma ISO 15213:2003. Organizzazione internazionale per la normazione ed., Ginevra, Svizzera.

ISO, 2004a. Microbiology of food and animal feeding stuffs. Horizontal method for the detection of Salmonella spp. Technical corrigendum 1: 2004. Norma ISO 6579: 2002/Cor 1:2004. Organizzazione internazionale per la normazione ed., Ginevra, Svizzera.

ISO, 2004b. Microbiology of food and animal feeding stuffs. Horizontal method for the enumeration of presumptive Bacillus cereus. Colony-count technique at 30 degrees C. Norma ISO 7932:2004. Organizzazione internazionale per la normazione ed., Ginevra, Svizzera.

ISO, 2004c. Modification of the enumeration medium. Norma ISO 11290-2:1998/Amd 1:2004. Organizzazione internazionale per la normazione ed., Ginevra, Svizzera.

ISO, 2005. Foodstuffs. Methods of analysis for the detection of genetically modified organisms and derived products. Nucleic acid extraction. Norma ISO 21571:2005. Organizzazione internazionale per la normazione ed., Ginevra, Svizzera.

ISO, 2006. Microbiology of food and animal feeding stuffs. Horizontal method for the enumeration of coliforms. Colony-count technique. Norma ISO 4832:2006. Organizzazione internazionale per la normazione ed., Ginevra, Svizzera.

ISO, 2007a. Microbiology of food and animal feeding stuffs. General requirements and guidance for microbiological examinations. Norma ISO 7218:2007. Organizzazione internazionale per la normazione ed., Ginevra, Svizzera.

ISO, 2007b. Microbiology of food and animal feeding stuffs. Horizontal method for the detection of potentially enteropathogenic Vibrio spp. Part 1: detection of Vibrio parahaemolyticus and Vibrio cholerae. Norma ISO/TS 21872:2007. Organizzazione internazionale per la normazione ed., Ginevra, Svizzera.

Istituto Superiore di Sanità, 1996. Rapporto ISTISAN 96/34. Istituto Superiore di Sanità ed., Roma. Disponibile al sito: http://www. iss.it/publ/rapp/cont.php?id=1758\&tipo $=5$ \&lang $=1 \&$ ccss $=000$

Marriott NG, Gravani RB, 2008. Sanificazione nell'industria alimentare. Springer Verlag Italia, Milano, Italia.

Ministero della Salute, 1971. Decisione del 14 dicembre 1971 concernente il limite di contaminazione da mercurio del pesce e degli altri prodotti alimentari della pesca di provenienza estera, D.M. 14/12/1971 $\mathrm{n}^{\circ}$ 328. In: Gazzetta Ufficiale, 28/12/1971.

Ministero della Salute, 1996. Decisione del 27 febbraio 1996 concernente la disciplina degli additivi alimentari consentiti nella preparazione e per la conservazione delle sostanze alimentari, in attuazione delle direttive n. 96/83/CE e n. 96/85/CE e della decisione n. 292/97/CE, D.M. 27/02/1996 n ${ }^{\circ}$ 
209. In: Gazzetta Ufficiale, $n^{\circ} 96$.

Ministero della Salute, 2010. Linee di indirizzo nazionale per la ristorazione scolastica. Ministero della Salute ed., Roma, Italia. Disponibile al sito: http:/ww.salute.gov.it/ imgs/c_17_pubblicazioni_1248_allegato.pdf Ministero della Salute, 2011. Relazione sullo stato sanitario del paese 2009-2010. Ministero della Salute ed., Roma, Italia. Disponibile al sito: www.salute.gov.it/ imgs/C_17_pubblicazioni_1655_allegato.pdf 0'Brien SJ, 2012. The public health impact of food-related illness. Curr Opin Infect Dis 25:537-45.

Repubblica Italiana, 1999. Attuazione delle direttive 96/22/CE e 96/23/CE concernenti il divieto di utilizzazione di talune sostanze ad azione ormonica, tireostatica e delle sostanze (beta)-agoniste nelle produzioni di animali e le misure di controllo su talune sostanze e sui loro residui negli animali vivi e nei loro prodotti. DL 4/08/1999, no. 336. In: Gazzetta Ufficiale no. 230, 30/09/1999.
Repubblica Italiana, 2007. Attuazione della direttiva 2004/41/CE relativa ai controlli in materia di sicurezza alimentare e applicazione dei regolamenti comunitari nel medesimo settore. DL 6/11/2007, no. 193. In: Gazzetta Ufficiale no. 261, 09/11/2007.

Schaffner DW, 2008. Microbial risk analysis of foods. ASM Press, Washington, D.C., USA.

Zacconi C, Vescovo M, Scolari G, 2001. Sicurezza igienico-sanitaria nella ristorazione collettiva. Ind Aliment-Italy 40:759 62 . 